\title{
Immunological characterization and transcription profiling of peripheral blood (PB) monocytes in children with autism spectrum disorders (ASD) and specific polysaccharide antibody deficiency (SPAD): case study
}

Harumi Jyonouchi ${ }^{1,2^{*}}$, Lee Geng ${ }^{1}$, Deanna L Streck ${ }^{2}$ and Gokce A Toruner ${ }^{2}$

\begin{abstract}
Introduction: There exists a small subset of children with autism spectrum disorders (ASD) characterized by fluctuating behavioral symptoms and cognitive skills following immune insults. Some of these children also exhibit specific polysaccharide antibody deficiency (SPAD), resulting in frequent infection caused by encapsulated organisms, and they often require supplemental intravenous immunoglobulin (IVIG) (ASD/SPAD). This study assessed whether these ASD/SPAD children have distinct immunological findings in comparison with ASD/nonSPAD or non-ASD/SPAD children.
\end{abstract}

Case description: We describe 8 ASD/SPAD children with worsening behavioral symptoms/cognitive skills that are triggered by immune insults. These ASD/SPAD children exhibited delayed type food allergy (5/8), treatment-resistant seizure disorders (4/8), and chronic gastrointestinal (Gl) symptoms (5/8) at high frequencies. Control subjects included ASD children without SPAD $(N=39)$, normal controls $(N=37)$, and non-ASD children with SPAD $(N=12)$.

Discussion and Evaluation: We assessed their innate and adaptive immune responses, by measuring the production of pro-inflammatory and counter-regulatory cytokines by peripheral blood mononuclear cells (PBMCs) in responses to agonists of toll like receptors (TLR), stimuli of innate immunity, and T cell stimulants. Transcription profiling of PB monocytes was also assessed. ASD/SPAD PBMCs produced less proinflammatory cytokines with agonists of TLR7/8 (IL-6, IL-23), TLR2/6 (IL-6), TLR4 (IL-12p40), and without stimuli (IL-1ß, IL-6, and TNF- $\alpha$ ) than normal controls. In addition, cytokine production of ASD/SPAD PBMCs in response to T cell mitogens (IFN- $\gamma$, IL-17, and IL-12p40) and candida antigen (Ag) (IL-10, IL-12p40) were less than normal controls. ASD/non-SPAD PBMDs revealed similar results as normal controls, while non-ASD/SPAD PBMCs revealed lower production of IL-6, IL-10 and IL-23 with a TLR4 agonist. Only common features observed between ASD/SPAD and non-ASD/SPAD children is lower IL-10 production in the absence of stimuli. Transcription profiling of PB monocytes revealed over a 2-fold up (830 and 1250) and down (653 and 1235) regulation of genes in ASD/SPAD children, as compared to normal $(\mathrm{N}=$ 26) and ASD/non-SPAD $(N=29)$ controls, respectively. Enriched gene expression of TGFBR $(p<0.005)$, Notch $(p<$ 0.01 ), and EGFR1 $(p<0.02)$ pathways was found in the ASD/SPAD monocytes as compared to ASD/non-SPAD controls.

Conclusions: The Immunological findings in the ASD/SPAD children who exhibit fluctuating behavioral symptoms and cognitive skills cannot be solely attributed to SPAD. Instead, these findings may be more specific for

\footnotetext{
* Correspondence: jyanouha@umdnj.edu

'Division of Allergy/Immunology and Infectious Diseases, Department of pediatrics, UMDN-NJMS, 185 South Orange Ave. Newark, NJ 07101-1709, USA Full list of author information is available at the end of the article
}

(C) 2012 Jyonouchi et al; licensee BioMed Central Ltd. This is an Open Access article distributed under the terms of the Creative 
ASD/SPAD children with the above-described clinical characteristics, indicating a possible role of these immune abnormalities in their neuropsychiatric symptoms.

Keywords: autism spectrum disorders (ASD), cytokine, innate immunity, transcription profiling, monocytes, specific polysaccharide antibody deficiency (SPAD)

\section{Background}

Mounting evidence indicate that ASD is a behaviorally defined syndrome associated with multiple genetic and environmental factors, resulting in similar behavioral symptoms [1-4]. The exceptions are small subsets of patients with known gene mutations (up to 15-20\%) [5]. Consequently, ASD is characterized by varying clinical phenotypes and a high frequency of co-morbidities. These co-morbid conditions often have inflammatory components and inflammation and immune activation has been implicated in ASD pathogenesis [6,7]. However, previous studies addressing immune abnormalities in ASD children have been inconclusive, partly due to the marked heterogeneity of the study subjects.

Previously, we reported a subset of ASD children whose clinical symptoms are characterized by worsening behavioral symptoms and loss of once acquired cognitive skills triggered by benign immune insults, typically common childhood infection [8]. Among this subset of ASD children, designated as the ASD-test group in the previous study, we found a high frequency of immunodeficiency (mainly SPAD), requiring treatment of intravenous immunoglobulin (IVIG) [8]. SPAD is clinically characterized by impaired antibody production against encapsulated organisms that are common causes of pneumonia, sinusitis, and ear infection. Therefore, in the previous study, ASD/SPAD children were excluded from the further analysis, due to the concern that the presence of SPAD and resultant presence of active infection may affect the results of our immunological assays. Thus, we do not know whether ASD/SPAD children with fluctuations in behavioral symptoms/cognitive skills have the innate immune abnormalities observed in the ASD test group [8] or if they manifest immune abnormalities more specific for SPAD.

In the Pediatric Allergy/Immunology (A/I) Clinic at our institution, we follow $8 \mathrm{ASD} / \mathrm{SPAD}$ children who have worsening behavioral symptoms/cognitive skills with immune insults. In these ASD/SPAD children, even after improved control of infectious complications with IVIG, we still observe worsening behavioral symptoms/cognitive skills that are triggered by immune insults. These children also seem to have treatment-resistant seizure disorders at a higher frequency than the ASD/non-SPAD children. In our observation, there were no differences between ASD/ SPAD children and non-ASD children with SPAD (nonASD/SPAD) in the routine immune workups. Infectious complications observed in these ASD/SPAD children in our clinic were very similar to those observed in nonASD/SPAD children [9]. Innate immune responses are not routinely studied in conventional immune workups for SPAD. Since our previous studies have indicated innate immune abnormalities in the ASD test group children [8], we hypothesized that innate immune responses affecting the development of adaptive cellular and humoral immunity are altered in the ASD/SPAD children who reveal worsening behavioral symptoms and cognitive skills with immune insults. We also hypothesized that these altered immune responses are not attributed to SPAD but are associated with their characteristic neuropsychiatric symptoms as described above, perhaps reflecting impaired neuroimmune network.

In this study, we tested our hypotheses by further characterizing $8 \mathrm{ASD} / \mathrm{SPAD}$ children with fluctuating behavioral symptoms/cognitive skills, by analyzing their clinical features and immunological findings in comparison with three control groups: ASD/non-SPAD children, normal control children, and non-ASD/SPAD children. The obtained results support our initial hypothesis, that peripheral blood mononuclear cells (PBMCs) from ASD/ SPAD children reveal distinct innate and adaptive immune abnormalities not shared by ASD/non-SPAD or non-ASD/SPAD children.

\section{Case description \\ ASD/SPAD children}

Eight ASD/SPAD children characterized by fluctuating (worsening) behavioral/cognitive skills following immune insults including viral infection and adverse reactions to medications were presented in this study. The demographics, diagnosis, co-morbidities, and clinical laboratory findings of these ASD/SPAD children are summarized in Tables 1 and 2. These ASD/SPAD children had at least 3 occurrences of worsening behavioral symptoms and/or loss of once acquired cognitive skills documented following immune insults such as viral syndrome; viral syndrome (upper respiratory infection and acute gastroenteritis) was clinically diagnosed with negative streptococcal antigen in throat swab and in some cases, supported by positive viral antigen and/or DNA in nasal secretions by PCR. Occurrences of worsening behavioral symptoms were independently documented by caretakers, teachers, and therapists. SPAD was diagnosed with detectable antibody $(\mathrm{Ab})$ titers $(>1.0 \mu \mathrm{g} / \mathrm{ml}$ ) to less than 3 of 14 serotypes of Streptococcus pneumonia tested in response to Pneumovax ${ }^{\circledR}[10]$, a 
Table 1 Demographics and clinical features of the ASD/SPAD children

\begin{tabular}{|c|c|c|c|c|c|c|c|}
\hline Case & $\begin{array}{l}\text { Age }^{1} \\
(y r)\end{array}$ & Race & Sex & $\begin{array}{l}\text { Immuno-deficiency } \\
\text { Diagnosis }\end{array}$ & $\begin{array}{l}\text { Autism } \\
\text { Diagnosis }\end{array}$ & Infection & Other co-morbidities and medications ${ }^{3}$ \\
\hline$\# 1^{4}$ & 13 & W & M & $\mathrm{SPAD}^{5}$ & Regressive autism & $\begin{array}{l}\text { CRS, } \\
\text { ROM }\end{array}$ & $\begin{array}{l}\text { Chronic enterocolitis, asthma } \\
\text { fluoxetine, montelukast, }\end{array}$ \\
\hline$\# 2^{4}$ & 11 & W & M & SPAD & Regressive autism & $\begin{array}{l}\text { CRS, } \\
\text { ROM }\end{array}$ & $\begin{array}{l}\text { Seizure disorder }{ }^{7} \text {, Chronic enterocolitis } \\
\text { levetiracetam, montelukast, loratadine, }\end{array}$ \\
\hline$\# 3^{4}$ & 8 & W & M & SPAD & Regressive autism & CRS & $\begin{array}{l}\text { Chronic enterocolitis, allergic rhiniconjunctivitis } \\
\text { fluoxetine, montelukast, cetirizine, mometasone nasal } \\
\text { inhaler }\end{array}$ \\
\hline$\# 4^{4}$ & 9 & W & M & SPAD & $\begin{array}{l}\text { PDD-NOS } \\
\text { (regressive) }\end{array}$ & ROM & $\begin{array}{l}\text { Seizure disorder } \\
\text { valproic acid, L-carnitine, CQ10 }\end{array}$ \\
\hline$\# 5^{2,4}$ & 14 & W & $\mathrm{F}$ & SPAD & Regressive autism & $\begin{array}{l}\text { CRS, } \\
\text { ROM, }\end{array}$ & $\begin{array}{l}\text { Seizure disorder, Chronic enterocolitis, asthma } \\
\text { montelukast, desloratadine, minocycline (for acne), } \\
\text { lorazepam }\end{array}$ \\
\hline \#6 & 16 & W & M & SPAD & Regressive autism & CRS & $\begin{array}{l}\text { Asthma, chronic enterocolitis } \\
\text { Steroid oral inhaler, nasal inhaler, guanfacine }\end{array}$ \\
\hline \#7 & 7 & W & $\mathrm{F}$ & SPAD & $\begin{array}{l}\text { PDD-NOS } \\
\text { (regressive) }\end{array}$ & $\begin{array}{l}\text { CRS, } \\
\text { ROM }\end{array}$ & $\begin{array}{l}\text { Seizure disorder } \\
\text { montelukast, nasal inhaler, levetiracetam, azithromycin } \\
\text { (prophylaxis) }\end{array}$ \\
\hline$\# 8$ & 6 & mixed & $M$ & $\mathrm{SPAD}^{6}$ & Regressive autism & $\mathrm{COM}$ & guanfacine, risperidone, benzatropine \\
\hline
\end{tabular}

${ }^{1}$ Ages at the time of SPAD diagnosis. It should be noted that Case \#1 and Case \#2 were followed up in the clinic for 2-3 yrs prior to SPAD diagnosis but their initial laboratory values were not consistent with SPAD diagnosis. Their clinical features progressed over 2-3 yrs to fulfill the diagnosis of SPAD.

${ }^{2}$ This patient developed anti-phospholipid syndrome 5 yrs after being treated with IVIG.

${ }^{3}$ Co-morbidities present at the time of presentation and medications at the time of sample obtainment.

${ }^{4}$ Positive history of food protein induced enterocolitis syndrome (FPIES)

${ }^{5}$ Abbreviations used: COM (chronic otitis media), CRS (chronic rhinosinusitis), PDD-NOS (pervasive developmental disorder, not otherwise specified), ROM (recurrent otitis media), SPAD (specific polysaccharide deficiency), and W (Caucasians)

${ }^{6}$ This patient also revealed low lgG levels but did not fall into the diagnostic criteria for common variable immunodeficiency; immunoglobulin levels of 2 isotypes are lower than two standard deviations of mean values of age-appropriate controls.

${ }^{7}$ In Case \#2 and Case \#7, a main trigger of seizure activities has been respiratory infection. In Case\#7, onset of seizure clusters were almost always triggered by respiratory infection prior to IVIG treatment. After implementation of IVIG treatment, no clinical seizures have been observed in case \#2. In case \#7, seizure activity appears to be not associated with infection any more after starting IVIG treatment and prophylaxis doses of azithromycin ( 3 times per week).

standard diagnostic measure for SPAD. All of the ASD/ SPAD children evaluated in this study are currently on IVIG $(0.6-1 \mathrm{~g} / \mathrm{kg} /$ dose every 3 weeks), since their infection complications were not controlled effectively with prophylactic antibiosis. Assay samples were obtained when their infectious complications were well under control after implementation of IVIG treatment. The ages of ASD/ SPAD children at the time of sample obtainment were $12.3 \mathrm{yr}$ (median) with range of 8.3-17.5 yr. The length of IVIG treatment varied from 1 to 6 yrs, at the time blood

Table 2 Summary of conventional immune workup results in ASD/SPAD children

\begin{tabular}{|c|c|c|c|c|c|c|}
\hline Case\# & $\operatorname{lgG}^{1}(\mathrm{mg} / \mathrm{dL})$ & $\lg A(m g / d L)$ & $\lg M(\mathrm{mg} / \mathrm{dL})$ & $\begin{array}{l}\text { Pneumococcal } \\
\text { antibody titers } \\
N>11 / 14\end{array}$ & $\begin{array}{l}\text { Isotype-switched memory B cells } \\
\quad(\text { cells } / \mu \mathrm{ll})^{3}\end{array}$ & $\begin{array}{c}\begin{array}{c}\text { Total memory B cells } \\
\text { (cells/ } \mu \mathrm{l})\end{array} \\
N=77.5(55.1-198)^{4}\end{array}$ \\
\hline$\# 1$ & 545 & 23 & 96 & $0 / 14$ & 7.6 & 19.6 \\
\hline$\# 2$ & 844 & 116 & 190 & $0 / 14$ & 8.3 & 13.2 \\
\hline$\# 3$ & 680 & 35 & 23 & $0 / 14$ & 6.1 & 15.1 \\
\hline$\# 4$ & 767 & 34 & 59 & $1 / 14$ & 5.4 & 25.0 \\
\hline$\# 5$ & 578 & 121 & 136 & $0 / 14$ & 2.7 & 2.9 \\
\hline$\# 6$ & 783 & 40 & 107 & $0 / 14$ & 21.9 & 31.0 \\
\hline$\# 7$ & 700 & 38 & 29 & $2 / 14$ & 8.0 & 40.8 \\
\hline$\# 8$ & 442 & 44 & 66 & $2 / 14$ & 1.9 & 19.0 \\
\hline
\end{tabular}

${ }^{1}$ Normal ranges of Ig levels $(\mathrm{mg} / \mathrm{dl})$ are for children of 6-8 yr (lgG; 572-1374, IgM; 30-208, IgA; 34-305) for children of 9-11 yr (IgG; 586-1496, IgM; 48-228, IgA; 45305 ) and for children of 12-16 yr (IgG 759-1549, IgM; 35-239, IgA; 58-358).

${ }^{2}$ Numbers of serotypes revealed $>1.0 \mu \mathrm{g} / \mathrm{ml}$ Ab levels among Streptococcus pneumonia 14 serotypes tested. Children $>5 \mathrm{yr}$ is expected to reveal protective levels of $A b$ titers $>75 \%$ of 14 serotype.

${ }^{3}$ Isotype switched memory B cell numbers identified as $\operatorname{lgD} D^{-}, C D 27^{+}, C D 19^{+}$cells and total memory B cell numbers (CD27 ${ }^{+}, \mathrm{CD} 19^{+}$cells) were expressed as cell numbers $/ \mu$ l of peripheral blood.

${ }^{4}$ Reference values of these parameters obtained from 27 normal controls (Age; 2-17 yr) in our laboratory are shown as a median (range). 
samples were obtained. It should be noted that we also follow 2 ASD/SPAD children without fluctuation in behavioral symptoms/cognitive skills. They responded very well to IVIG treatment for controlling infections but we did not observe any changes in their autistic features. These children were not included in the current study. We refer ASD/SPAD children as these 8 ASD/SPAD children with worsening behavioral symptoms and cognitive skills with immune insults in this study.

In both ASD/SPAD and ASD/non-SPAD children, autism diagnosis was from established autism diagnostic centers, including the center at our institution. Standard diagnostic measures, including ADOS (Autism Diagnostic Observational Schedules), and ADI-R (Autism Diagnostic Interview-Revised) were used. Diagnosis of allergic disorders and asthma were based on diagnostic criteria described elsewhere [11-13].

\section{Control subjects}

1. ASD/non-SPAD children: These children were recruited in the Subspecialty Clinic at UMDNJ-NJMS where subspecialties include allergy/immunology, cardiology, developmental pediatrics, endocrinology, gastroenterology, genetics, general pediatrics, nephrology, and pulmonology. A total of 39 ASD/non-SPAD children were recruited to the study: 4 females and 35 males, median age: $8.1 \mathrm{yr}$, range; 5-17 yr, 7 African Americans (AA), 3 Asians, 27 Caucasians (W), and 2 mixed races. These ASD/nonSPAD children were diagnosed with autism $(\mathrm{N}=25)$ or PDD-NOS $(\mathrm{N}=14)$. Twenty out of 39 children reported to have developmental regression at the time of initial diagnosis of ASD. Allergic rhinoconjunctivitis $(\mathrm{AR}+\mathrm{AC})$ was diagnosed in 5/39 (12.8\%) ASD/non-SPAD children. None of the control ASD/non-SPAD children were documented to have recurrent infection and/or fluctuating behavioral symptoms/cognitive skills following immune insults. In addition, none of these ASD/non-SPAD controls were diagnosed with seizure disorders.

2. Normal controls: Normal control children $(\mathrm{N}=37,8$ females and 29 males, median age; $10.2 \mathrm{yr}$, range: 5-17 yr, 4AA, 1 Asian, $31 \mathrm{~W}$, and 1 mixed race) were recruited in the Pediatric Subspecialty Clinic. In most cases, blood samples were obtained when they were medically indicated to have venipuncture for general health screening.

3. Non-ASD/SPAD children: A total of 12 non-ASD/ SPAD children were recruited in the pediatric Allergy/ Immunology Clinic; median age; $13.0 \mathrm{yr}$, range; 6-17 yr, 6 females and 6 males, $4 \mathrm{AA}$ and $8 \mathrm{~W}$. SPAD diagnosis was made as described in ASD/SPAD children in the previous section [10]. All of them have been treated with IVIG (0.6$1 \mathrm{~g} / \mathrm{kg} /$ dose every 3 weeks) and were on IVIG treatment at the time of sample obtainment. The length of IVIG treatment was 1 to 7 yrs for these children at the time of this study. Two out of 12 patients were diagnosed with seizure disorders; in 1 patient, the seizure disorder was attributed to sequel of intracranial abscess developed from chronic rhinosinusitis and in 1 patient, the etiology of seizure disorder is unknown.

Sample obtainment The study subjects were recruited following study protocols approved by the Institutional Review Board, University of Medicine and Dentistry of New Jersey-New Jersey Medical School (UMDNJ-NJMS). Blood samples were collected after obtainment of signed parental consent forms. Signed assent forms were also obtained, if applicable, in children older than 7 years of age.

At the time of sample obtainment, all the subjects were examined to ensure absence of active infection. Assays for adaptive and innate immunity for SPAD children, with or without ASD, were conducted after their conditions were stabilized by IVIG and became free from active infection. In most cases, sample obtainment coincided with medically indicated blood work.

Clinical and laboratory findings in ASD/SPAD children by conventional immune workup Most of the ASD/SPAD children suffered from chronic rhinosinusitis (CRS) and recurrent otitis media (ROM) requiring frequent antibiosis prior to IVIG treatment. Four out of 8 (50\%) ASD/SPAD children had history of multiple placements of pressure equalizing $(\mathrm{PE})$ tubes bilaterally (Table 1). ASD/SPAD children also revealed a high frequency of treatment-resistant seizure disorders $(4 / 8,50 \%)$, while none of the normal and ASD/non-SPAD control children had a history of seizure disorders. However, 25/40 (62.5\%) ASD/non-SPAD children had a history of food protein induced enterocolitis syndrome (FPIES), although at the time of blood sampling, none of them had active GI symptoms. ASD/SPAD children also had history of FPIES at a similar rate $(5 / 8,62.5 \%)$ and these ASD/SPAD children with history of FPIES suffered from chronic enterocolitis, even after IVIG treatment, requiring dietary intervention measures (avoidance of offending food). In these ASD/ SPAD children with GI symptoms, extensive workups ruled out chronic microbial infection, celiac disease, inflammatory bowel diseases, or other well established GI diseases by endoscopic and histological examinations. None of the ASD/SPAD subjects revealed positive allergy workups; they all had normal IgE levels, negative reactivity to prick skin testing (PST), or negative for food allergen specific IgE. All the ASD/SPAD children were diagnosed with regressive type ASD (Table 1).

As shown in Table 2, immune workups, at initial diagnosis of SPAD, revealed low to low normal serum levels of IgG, IgA, and IgM as compared to age-appropriate controls in 4/8,6/8, and 6/8 ASD/SPAD children. Numbers of isotype-switched memory B cell were lower than $10 / \mu$ in 7/8 ASD/SPAD children [14]. Infection was better controlled after initiation of IVIG treatment in all the ASD/ SPAD children, which was also associated with a reduction 
in frequency of worsening behavioral symptoms triggered by infection. After being treated with several doses of IVIG, the parents of one patient reported the return of cognitive skills that were present prior to major regression. However, 7/8 ASD/SPAD children did not reveal significant improvement in autism behavioral symptoms or cognitive activity, per parental reports assessed by the clinical global impression scale (CGI) [15]. In the ASD/SPAD patients with seizure disorders, frequency of seizures was reduced after starting IVIG treatment, which is likely attributed to better control of infection, since the major trigger of seizure activity is infection for these subjects.

\section{Further Evaluation of immune functions}

1. PBMC Cultures PBMCs were isolated by Ficoll-Hypaque density gradient centrifugation. Innate immune responses were assessed by incubating PBMCs $\left(10^{6}\right.$ cells/ $\mathrm{ml}$ ) overnight with TLR4 agonist (LPS; $0.1 \mu \mathrm{g} / \mathrm{ml}$, GIBCO-BRL, Gaithersburg, MD), TLR2/6 agonist (zymosan; $50 \mu \mathrm{g} / \mathrm{ml}$, Sigma-Aldrich, St. Luis, Mo), TLR3 agonist (Poly I:C, Poly I:C, $0.1 \mu \mathrm{g} / \mathrm{ml}$, Sigma-Aldrich), and TLR7/8 agonist (CL097, water-soluble derivative of imidazoquinoline, $20 \mu \mathrm{M}$, InvivoGen, San Diego, CA) in RPMI 1640 with additives as previously described [8]. Overnight incubation was adequate to induce the optimal responses in this setting. Levels of proinflammatory [tumor necrosis factor- $\alpha$ (TNF- $\alpha$ ), IL-1 $\beta$, IL-6, IL-12p40, and IL-23] and counter-regulatory [IL-10, transforming growth factor- $\beta$ (TGF- $ß$ ) and soluble TNF receptor II (sTNFRII)] cytokines in culture supernatant were then measured by an enzyme-linked immunosorbent assay (ELISA).

Cellular reactivity to $\mathrm{T}$ cell stimulants was assessed by incubating PBMCs $\left(10^{6}\right.$ cells $\left./ \mathrm{ml}\right)$ with $\mathrm{T}$ cell mitogens [Con A $(2 \mu \mathrm{g} / \mathrm{ml})$ and PHA $(5 \mu \mathrm{g} / \mathrm{ml})$ ], recall Ag [candida $\mathrm{Ag}(5 \mu \mathrm{g} / \mathrm{ml})$, Greer, Lenoir, NC], and IFN- $\gamma$ inducing cytokines [IL-12p70 (0.2 ng/ml, BD Biosciences, San Diego, CA), IL-18 (1 ng/ml, BD Biosciences) for 4 days and measuring levels of IFN- $\gamma$, TNF- $\alpha$, IL-5, IL-10, IL-12p40, and IL-17 in the culture supernatant [8]. Initial titration studies showed that a four-day incubation period resulted in optimal production of these cytokines, in this setting.

Cytokine levels were measured by ELISA, using OptEIA ${ }^{\mathrm{TM}}$ Reagent Sets (BD Biosciences) for IFN- $\gamma$, IL-1ß, IL-5, IL-6, IL-10, IL-12p40, and TNF- $\alpha$, and ELISA reagent set ( $\mathrm{R} \& \mathrm{D}$, Minneapolis, $\mathrm{MN}$ ) for sTNFRII, IL-17 (IL-17A), and TGF-ß. IL-23 ELISA kit was purchased from eBiosciences, San Diego, CA. Intra- and inter-variations of cytokine levels were less than $5 \%$.

2. Flow cytometry Memory B cells (IgD ${ }^{-}, \mathrm{CD} 27^{+}, \mathrm{CD} 19^{+}$ $B$ cells) were detected by staining with anti-CD45-FITC, anti-CD19-APC-Cy7, CD27-APC (all from BD biosciences, San Jose CA) and IgD-PE (DAKO, Carpinteria CA) monoclonal antibodies [14]. For intracellular cytokine staining in $\mathrm{CD}^{+} \mathrm{T}$ cells, the following fluorochrome-conjugated monoclonal antibodies were used: CD4-PerCp, IFN- $\gamma$-PE-Cy7, IL-17-PE, IL-4-FITC, IL-10Pacific Blue (all from eBiosicences), and TGF-ß-APC ( $\mathrm{R} \& \mathrm{D}$, Minneapolis, MN). PBMCs were incubated overnight $(16 \mathrm{~h})$ at $37^{\circ} \mathrm{C}$ with medium alone, Staphylococcal enterotoxin B $(5 \mu \mathrm{g} / \mathrm{mL}$, Sigma-Aldrich), or candida Ag $(5 \mu \mathrm{g} / \mathrm{ml}$, Greer) in the presence of Brefeldin A (BFA; 5 $\mu \mathrm{g} / \mathrm{ml}$, Sigma-Aldrich), anti-CD28 (1 $\mu \mathrm{g} / \mathrm{ml}$, eBiosciences), and anti-CD49 (1 $\mathrm{gg} / \mathrm{ml}$, eBiosciences) in the same culture medium used for the cytokine production assay. Then PBMCs were permeabilized (permeabilization buffer, BD Biosciences) and stained with the above described antibodies [16]. All flow cytometry was conducted using FACS Caliber or FACSVantage SE TM (BD Biosciences) and the data were analyzed with the CellQuest software (BD Biosciences) and FlowJo (TreeStar, Ashland, OR).

3. Transcription profiling Peripheral blood (PB) monocytes were purified using an immuno-affinity column following the manufacturer's instructions (MACS monocytes isolation kit, Miltenyi Biotec, Auburn, CA). Total RNA were extracted by the RNA easy kit (Quiagen, Valencia, CA). RNA labeling and hybridizations on Agilent Human $4 \times 44 \mathrm{~K}$ arrays (Agilent, Lexington, MA) were done using the Agilent One-Color Microarray-Based Gene Expression Analysis Ver 5.5 protocol (Agilent). All slides were scanned by an Agilent Scanner and normalized numerical data were obtained by Agilent Feature extraction software 9.5 .

4. Statistical analysis For comparison of test values with control values, a Wilcoxon rank sum test was used. For comparison of values of multiple groups, a KruskallWallis test was used. A Chi square $\left(\chi^{2}\right)$ test was used to examine the difference in frequency and correlation was tested using a linear regression analysis. These tests were performed using R.2.10.1 (R-Development Core Team 2009). A p value of $<0.05$ was considered to be statistically significant. For the analysis of microarrays experiments, Gene Spring GX v11 software (Agilent) was used. After filtering for "present" calls in at least $20 \%$ of samples, fold change analysis were performed for group for comparisons on 26992 probes. Genes with at least a twofold change, as compared to controls, are determined to be either up-regulated or down-regulated. Using a specific module of GeneSpring software (Agilent), pathways enrichment analysis was performed on those up- or down-regulated genes to see if there is a statistically significant enrichment $(\mathrm{p}<0.05)$ for specific BioPax pathways.

5. Cytokine production results As stated above, the median ages of the study subjects at the time of sample obtainment are $12.3 \mathrm{yr}$ for ASD/SPAD children, $8.1 \mathrm{yr}$ for ASD/non-SPAD children, 10.2 yr for normal controls, 
and $13.0 \mathrm{yr}$ for non-ASD/SPAD children. Ages of ASD/ non-SPAD children were lower than ASD/SPAD and non-ASD/SPAD children $(\mathrm{p}<0.05)$. It should be noted that innate immune responses, as opposed to adaptive immune responses, are not expected to change with age. This is mainly because innate immunity is regulated by germ-line coded genes and has little post-natal modifications, such as gene rearrangement.

Responses to TLR agonists

ASD/SPAD PBMCs produced different patterns of cytokine production. Namely, ASD/SPAD PBMCs produced lower amounts of IL- 6 (without a stimulus and with TLR2/6 agonists), IL-1ß (without a stimulus), and IL-23 (with TLR 7/8 agonist) as compared to all the control groups (Figure $1 \mathrm{~A}, \mathrm{~B}, \mathrm{C}$ ). In, addition, ASD/SPAD PBMCs produced less IL-12p40 than normal and nonASD/SPAD control cells in response to a TLR4 agonist (Figure 1-A). These cells also produced lower amounts of IL-6 (with the TLR 7/8 agonist) and TNF- $\alpha /$ IL-10 (in the absence of stimulus) than normal controls (Figure 1-
B). PBMCs from ASD/non-SPAD children revealed similar patterns of cytokine production as compared to normal controls (Figure 1). In contrast, non-ASD/SPAD PBMCs revealed altered patterns of cytokine production which did not resemble those observed in ASD/SPAD children. That is, non-ASD/SPAD PBMC revealed lower IL-10 production than ASD/SPAD and normal control cells (Figure 1-A) and lower IL-6/IL-23 production than normal controls (Figure 1-C, D) in response to a TLR4 agonist. The only common feature observed between the ASD/SPAD and non-ASD/SPAD groups was production of lower levels of IL-10 in the absence of a stimulus, than normal controls (Figure 1-C). These results indicate that the altered responses to TLR agonists observed in the ASD/SPAD group are unlikely to be associated with SPAD.

Responses to the recall antigen and $\mathrm{T}$ cell mitogens ASD/SPAD PBMCs also revealed altered patterns of $\mathrm{T}$ cell cytokine production as compared to control groups. Namely, ASD/SPAD PBMCs produced less IL-12p40

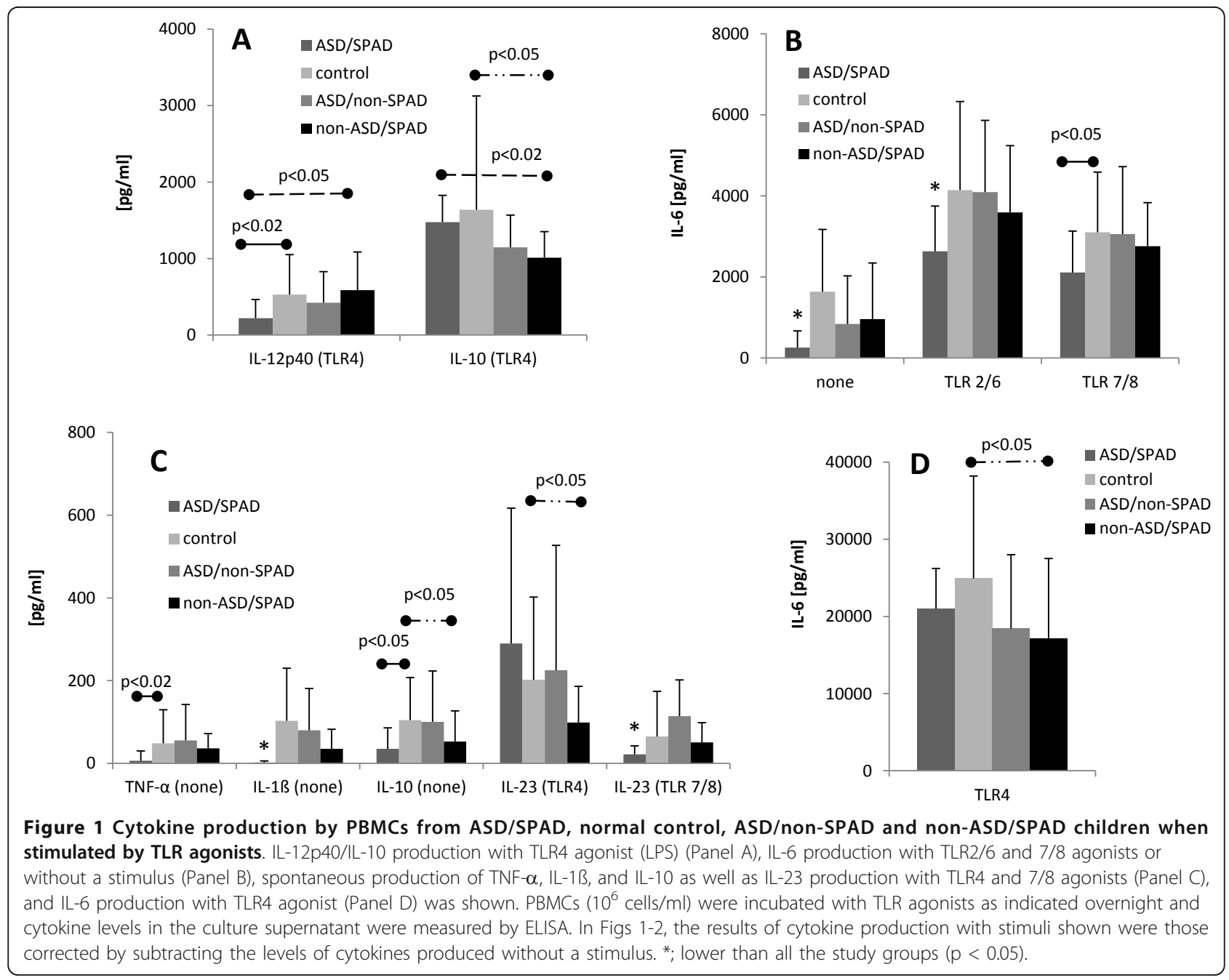


and IL-10 than all the study groups in response to a recall $\mathrm{Ag}$ (candida $\mathrm{Ag}$ ) (Figure 2-B). In addition, ASD/ SPAD PBMCs produced less IFN- $\gamma$ and IL-17A with PHA, and less IL-12 with Con A as compared to normal controls (Figure 2-A). Moreover, amounts of IL-17A produced by ASD/SPAD PBMCs with PHA was also lower than that of non-ASD/SPAD cells (Figure 2-A). IL12 production with Con A by ASD/SPAD cells was also lower than that of ASD/non-SPAD controls (Figure 2-A).
Production of T cell cytokines by ASD/non-SPAD and non-ASD/SPAD cells did not differ from normal controls in response to $\mathrm{T}$ cell mitogens or candida Ag (Figure 2-A \& 2 B). TGF- $B$ is produced in high amounts spontaneously and does not increase much in response to $\mathrm{T}$ cell stimuli. However, interestingly, we observed a higher increase in TGF- $\beta$ production in response to candida $\mathrm{Ag}$ in the ASD/SPAD group than normal and non-ASD/ SPAD groups. Again, these results indicate that altered

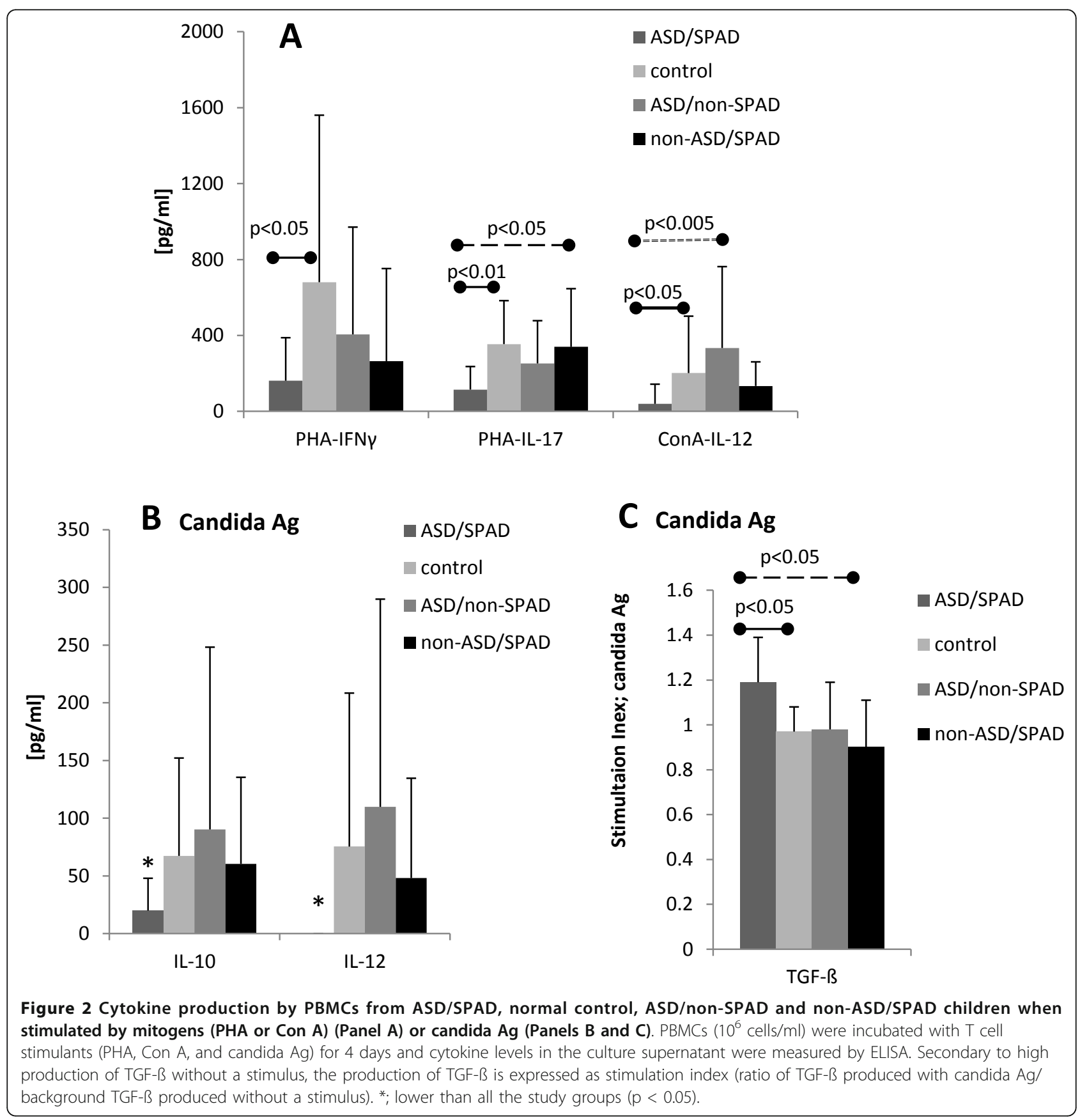


patterns of $\mathrm{T}$ cell cytokine production by the ASD/SPAD PBMCs is unlikely to be attributed to SPAD.

When intracellular expression of Th1 (IFN- $\gamma$ ), Th2 (IL-4), Th17 (IL-17A), and regulatory cytokines (IL-10 and TGF- 3 ) were examined in 6/8 ASD/SPAD children, we observed lower intracellular expression of TGF- $\beta$ in $\mathrm{CD}^{+}$cells, as compared to age-appropriate controls (ASD/non-SPAD children $\mathrm{N}=18$, normal controls $\mathrm{N}=$ 26, and non-ASD/SPAD children $\mathrm{N}=9$ ) (Figure 3).

6. Transcription profiling of PB monocytes Since most notable changes were found in TLR responses in ASD/ SPAD children and these changes were not observed in non-ASD/SPAD controls, we conducted transcription profiling of $\mathrm{PB}$ monocytes, a major cell population of $\mathrm{PB}$ innate immune cells responding to TLR agonists. Transcription profiling was conducted in 7 ASD/SPAD, 28 ASD/non-SPAD, and 26 normal control children. We were unsuccessful in 2 our attempts to purify total RNA from PB monocytes of 1 ASD/SPAD child; this may be associated with the high dose of valproic acid that he was on in order to control his seizure activities. Our results revealed that significant numbers of genes were either up or down-regulated (> 2 fold) in ASD/SPAD monocytes as compared to controls, as summarized in Table 3. Up-regulated genes as compared to control groups included chemokines (CCL2 and CCL7). Pathway analysis of gene expression profiles revealed that ASD/SPAD children had enriched expression of genes involved in TGFBR (TGF- $\beta$ receptor), EGFR (epidermal growth factor receptor), and $\mathrm{NOTCH}$ pathways as compared to ASD/non-SPAD controls (Table 3).

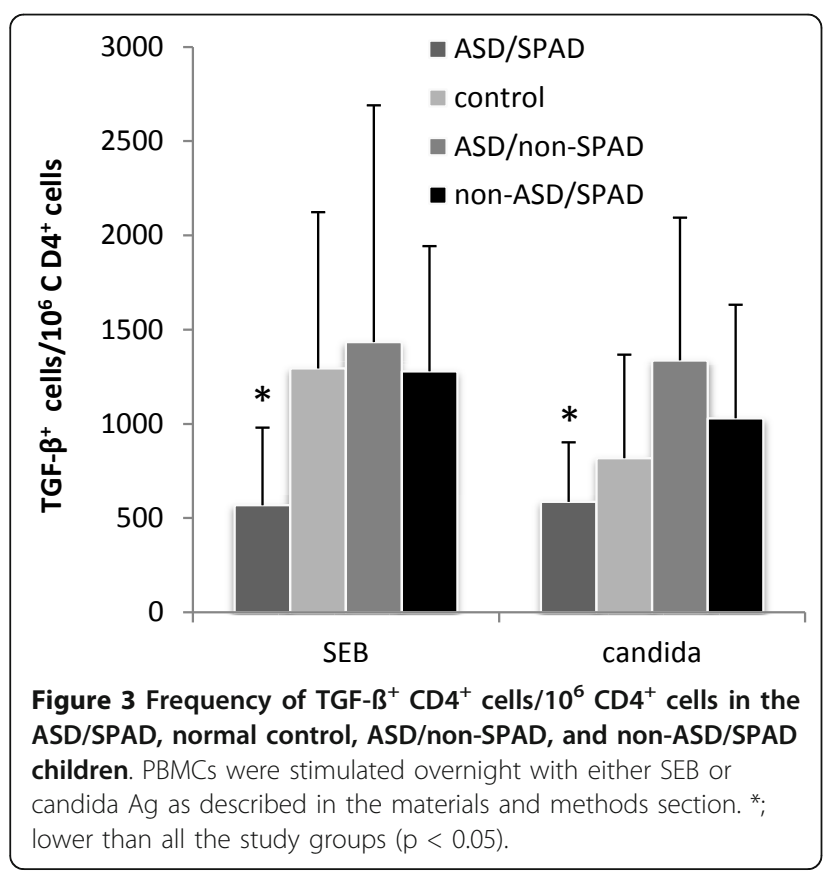

\section{Discussion and Evaluation}

Clinical features of infection found in ASD/SPAD children are similar to those found in non-ASD/SPAD children and all of the ASD/SPAD children suffered from frequent sinopulmonary infection (Table 2) [17]. As expected, infections were better controlled, once IVIG treatment was in place $[18,19]$. However, the ASD/SPAD children revealed a higher frequency of seizure disorders as compared to nonASD/SPAD children (4/8, 50\% vs. $2 / 1216.7 \%)$. In $2 / 4$ ASD/SPAD children with seizure disorders, seizure activity was often associated with infection and is better controlled after implementation of IVIG treatment (Table 1). No subjects in the normal control and ASD/non-SPAD groups suffered from seizure disorders. As noted previously, these ASD/SPAD subjects are those with markedly worsening behavioral symptoms/cognitive skills following each immune insult [8]. These results raise the question of whether the immune abnormalities that are associated with SPAD, contribute to the clinical features observed in the ASD/SPAD children. Alternatively, it may be possible that SPAD is a part of the clinical features that develop with age in the ASD/SPAD children and are associated with immune abnormalities that affect $\mathrm{Ab}$ production and possibly the neuroimmune network. Interestingly, in 3/4 ASD/SPAD subjects with seizure disorders, SPAD was diagnosed several years later after the diagnosis of seizure disorders.

Routine immune workups of ASD/SPAD children did not reveal major defects of $\mathrm{T}$ or $\mathrm{B}$ cells, except for those indicating impaired antibody production typically found in SPAD patients (Table 2). Infectious complications in the ASD/SPAD children were very similar to those observed in non-ASD/SAPD children [9]. Therefore, pathogen-specific mechanisms are unlikely to explain neuropsychiatric symptoms of fluctuating behavioral symptoms/cognitive skills in the ASD/SPAD children examined in this study.

When innate immune responses were assessed, by measuring responses to a panel of TLR agonists, we observed significant differences in the ASD/SPAD children, as compared to control groups. That is, PBMCs from the ASD/ SPAD children tended to produce less pro-inflammatory cytokines (TNF- $\alpha$, IL-1ß, IL-6, IL-12, and IL-23). Changes were most evident in the production of IL-6. As for production of counter-regulatory cytokines, PBMCs from ASD/SPAD children revealed less spontaneous IL-10 production than normal controls. Responses to TLR agonists in the non-ASD/SPAD children differed significantly from those observed in the ASD/SPAD children as detailed in the results section and Figure 1. Only common feature found in both the ASD/SPAD and non-ASD/SPAD children was lower spontaneous production of IL-10 than normal controls (Figure 1-C).

Since ASD/SPAD children are on multiple medications for asthma, chronic rhinitis, and infection 
Table 3 Summary of transcription profiling results in ASD/SPAD children

\begin{tabular}{llll}
\hline Numbers of genes ${ }^{\mathbf{1}}$ & Vs. normal controls & Vs. ASD/non-SPAD controls & Overlapping in 2 control groups \\
\hline Up-regulated & 830 & 1258 & 316 \\
\hline Down-regulated & 653 & 1235 & 356 \\
\hline Pathway analysis & Vs. normal controls & Vs. ASD/non-SPAD controls & \\
\hline TGFBR & Not significant & $P<0.01$ & \\
\hline EGFR & Not significant & $P<0.02$ & \\
\hline NOTCH & Not significant & $P<0.005$ & \\
\hline
\end{tabular}

${ }^{1}$ The number of genes $>2$ fold up- or down-regulated in the ASD/SPAD children as compared to normal controls and ASD/non-SPAD children.

prophylaxis which included montelukast, steroid oral/ nasal inhalers, anti-histamines, and azithromycin prophylaxis, these finding could be attributed to medications that they were on. However, non-ASD/SPAD children were also on multiple medications similar to those taken by ASD/SPAD children. Therefore it is unlikely that these medications are affecting the assay results. Some ASD/SPAD children were also on anti-seizure medications including levetiracetam $(\mathrm{N}=2)$, valproic acid $(\mathrm{N}=1)$, and lorazepam $(\mathrm{N}=1)$. However, it is hard to assess if these medications can affect the assay results given low frequency of intake of these medications among the ASD/SPAD children. Two ASD/ non-SPAD children with seizure disorders were also on levetiracetam and valproic acid.

IL-6 is important for B cell maturation and Ab production [16]. Our finding of impaired IL-6 production in ASD/SPAD children might be associated with development of SPAD in the ASD/SPAD children. Decreased production of IL-10 may also indicate a possibility of prolonged inflammation in ASD/SPAD children. Proinflammatory cytokines especially TNF- $\alpha$, IL- $1 ß$, and IL- 6 exert important roles in mediating acute stress responses and dysregulated production of these cytokines were implicated with chronic CNS inflammation [20,21], as well as, schizophrenia [22]. Thus, lower production of these key cytokines may indicate an impairment of stress responses or the neuro-immune network in the ASD/ SPAD children with fluctuating behavioral symptoms/cognitive skills.

Cytokines produced by innate immune responses greatly affect differentiation of T-helper (Th) cell subsets. IL-1ß, IL-6, and TGF- $\beta$, when combined together, promote differentiation of Th17 cells, which in turn promote neutrophilic inflammation and anti-fungal/bacterial defense [23-25]. IL-23 sustains Th17 cells [23-25]. IL-12 promotes differentiation of Th1 cells [26]. Given decreased IL-12, IL-6, and IL-1ß production in the ASD/ SPAD children, the question is raised as to whether production of $\mathrm{T}$ cell cytokines specific for Th cell subsets is altered in ASD/SPAD children. When we tested T cell cytokine production, our results revealed lower production of IFN- $\gamma$, Th1 cytokine, and IL-17A, Th17 cytokine, in response to PHA in the ASD/SPAD children (Figure 2-A). These results indicate a possible impairment of Th1 and perhaps Th17 responses in the ASD/SPAD children, making them more vulnerable to certain microbial infection. However, frequency of Th1 and Th17 subsets identified by intracellular cytokine expression were not altered in ASD/SPAD children. The non-ASD/SPAD children did not reveal such changes. Further studies regarding Th1/Th17 cell development will be required in the ASD/SPAD children with fluctuating behavioral symptoms and cognitive skills.

When we tested adaptive immune responses to recall Ags, we observed significantly less production of IL-10 and IL-12p40 with candida Ag, but higher increase of TGF- $\beta$ production in the ASD/SPAD children, than control groups. IFN- $\gamma$ or IL-17A production with candida Ag did not differ among the study groups. Interestingly, 5 of 8 ASD/SPAD children had chronic GI inflammation often complicated by dysbiosis and/or candida enteritis with evidence of positive reactivity to candida antigen when assessed by production of IFN- $\gamma$ and IL-17A production at the time of flare up. These children frequently required treatments with oral anti-fungal medications. Both IL-10 and IL-12p40 can function as regulatory factors to control inflammatory responses. Thus a decreased production of these cytokines with candida Ag may lead to persistent and excessive immune responses against candida Ag in the ASD/SPAD children.

Intracellular expression of TGF- $\beta$ was lower in the ASD/SPAD children than controls (Figure 7) when stimulated with SEB or candida Ag. These findings indicate decreased frequency of TGF- $\beta^{+}$inducible regulatory $\mathrm{T}$ (Treg) cells in the ASD/SPAD children, despite higher increase in TGF- $\beta$ production by ASD/SPAD PBMCs than controls. TGF- $\beta$ is produced by many lineage cells and the source of TGF- $\beta$ in the cultures of ASD/SPAD PBMCs may not be Treg cells, but other lineage cells. Such changes were not observed in non-ASD/SPAD children. In summary, studies of $\mathrm{T}$ cell functions indicate dysregulated $\mathrm{T}$ cell functions in the ASD/SPAD children, but not in the non-ASD/SPAD children. These findings may be associated with altered innate immune responses in the ASD/SPAD children. 
Circulating monocytes in the PB have a short half-life and undergo spontaneous apoptosis on a daily basis [27]. In response to various differentiation factors, monocytes escape their apoptotic fate by differentiating into macrophages $[27,28]$, which usually happens during inflammatory responses. PB monocytes are heterogeneous consisting of M1 monocytes (CD14++, CD16cells) and M2 monocytes (CD14+, CD16+ cells) [27,29]. The majority of PB monocytes is M1 monocytes and activated M1 monocytes are recruited to the site of inflammation via chemokines (CCL2 and CCL7) [27,29]. To further extend our investigations, we conducted transcription profiling of PB monocytes in ASD/SPAD children in comparison with ASD/non-SPAD and normal controls. Over 300 genes are either up- or downregulated in the $\mathrm{ASD} / \mathrm{SPAD}$ children, in comparison with both ASD/non-SPAD and normal control groups (Table 3). Interestingly, gene expression of CCL2 and CCL7 was up-regulated in PB monocytes from the ASD/SPAD children. Recruitment of M1 monocytes has been implicated with chronic inflammatory conditions, including those in the CNS [27,30]. Thus, up-regulated expression of CCL2 and CCL7 may indicate a constant activation signal occurring in $\mathrm{PB}$ monocytes in the ASD/SPAD children.

The results of transcription profiling of $\mathrm{PB}$ monocytes also revealed enriched expression of genes in TGFBR (TGF- $\beta$ receptor), NOTCH, and EGFR (epidermal growth factor receptor) signaling pathways in ASD/ SPAD children, as compared to ASD/non-SPAD controls. Pathogen associated molecular patterns (PAMPs) often up-regulate $\mathrm{NOTCH}$ ligands and $\mathrm{NOTCH}$ signaling in macrophage/monocyte lineage cells [31], which can, in turn, affect production of cytokines regulating Th cell subsets [32]. NOTCH signaling is also known to affect the activation status of microglial cells and neural progenitor cells [33-35]. NOTCH and EGFR pathways are closely inter-related and involved in regulation of cell differentiation and proliferation [36,37]. TGFBR pathway activation is implicated with cell proliferation and wound healing along with its anti-inflammatory properties of TGF- $ß$ [38]. In addition, TGF- $ß$ pathways closely interact with NOTCH and EGFR signaling pathways [31]. Thus the findings from the transcription profiling of $\mathrm{PB}$ monocytes also support our assumption that ASD/SPAD monocytes are chronically activated which may be associated with the fluctuating neuropsychiatric symptoms observed in the ASD/SPAD children. Further analysis of a larger number of these children in comparison with appropriate controls will be necessary for further elucidate whether $\mathrm{PB}$ monocytes play a role in the medical conditions of ASD/SPAD children. In addition, follow-up of these parameters in the ASD/SPAD children longitudinally will be informative to further address their immune abnormalities in association with their above-described clinical features.

Use of IVIG for treatment of autism or its 'presumed' autoimmune co-morbid conditions, such as pediatric autoimmune neuropsychiatric disorders associated with streptococci (PANDAS), has been controversial, and clinical trials yielded conflicting results [39-41]. The controversy surrounding the effects of IVIG on ASD children is partly attributed to marked heterogeneity of ASD subjects and relatively small numbers of study subjects. In our cohorts, beneficial effects of IVIG on cognitive skills/behavioral symptoms were observed in only one of 8 ASD/SPAD subjects, indicating the complexity of ASD pathogenesis and the need for careful evaluation of the use of IVIG in ASD children, other than for antibody deficiency syndrome.

The limitation of this study is the lack of transcription profiling data in $\mathrm{PB}$ monocytes from the non-ASD/ SPAD children, due to both limited resources and restrictions associated with the study protocol. However, to our knowledge, we have not found any literature describing similar changes in transcript profiles of $\mathrm{PB}$ monocytes in non-ASD/SPAD children. At least two ASD/SPAD children without fluctuation of behavioral symptoms/cognitive skills are followed in our clinic. In these children, we have not observed the immune abnormalities found in ASD/SPAD children described in this case series (unpublished observation). These results again indicate that the immune abnormalities observed in ASD/SPAD children with fluctuating behavioral symptoms/cognitive skills are not solely attributed to SPAD.

\section{Conclusions}

In summary, our results revealed distinct clinical and immunological findings in ASD/SPAD children who reveal worsening behavioral symptoms/cognitive skills with immune insults. These immune abnormalities are not shared by either ASD/non-SPAD or non-ASD/ SPAD children. Thus these abnormalities are likely to be more specific for this subset of ASD children and may be associated with their worsening behavioral symptoms/cognitive skills with immune insults. Development of SPAD in these ASD children may also be a part of their clinical spectrum associated with these immune abnormalities.

\section{Abbreviations}

Abbreviations used are as follows: AA: African American; Ab: antibody; Ag: antigen; ASD: autism spectrum disorders COM; chronic otitis media CRS: chronic rhinosinusitis; CVID: common variable immunodeficiency; FA: food allergy; FPIES: food protein induced enterocolitis syndrome; IVIG: intravenous immunoglobulin; IL: interleukin; PB: peripheral blood; PBMCs: peripheral blood mononuclear cells; PDD-NOS: pervasive developmental disorder, not otherwise specified; PE tube: pressure equalizing tube; SEB: staphylococcal 
enterotoxin B; SPAD: specific polysaccharide antibody deficiency; sTNFRII: soluble TNF receptor II; Th: T-helper; TGF: transforming growth factor; TNF: tumor necrosis factor.

\section{Acknowledgements}

The authors thank all the study subjects and parents for donating blood samples. This study was partly supported by a grant from Jonty Foundation, St. Paul, MN and Autism Research Institute, San Diego, CA. Authors are also thankful for Dr. L. Huguienin for critically reviewing this manuscript.

\section{Author details}

'Division of Allergy//mmunology and Infectious Diseases, Department of pediatrics, UMDN-NJMS, 185 South Orange Ave. Newark, NJ 07101-1709, USA. ${ }^{2}$ The Institute of Genomic Medicine, Department of Pediatrics, UMDNJNJMS185 South Orange Ave. Newark, NJ 07101-1709, USA.

\section{Authors' contributions}

HJ was responsible for the study design, recruitment of the study subject, collection of clinical information and blood samples, and analysis of the data of cytokine production assays and flow cytometry. She was also mostly responsible for preparation of this manuscript. LG conducted most of cytokine production assays as well as staining cells for flow cytometry and assisted the first author for data analysis. She was also responsible for PB monocytes purification and preparation of total RNA from PB monocytes. DLS was mostly responsible for conducting transcription profiling of PB monocytes.

GAT was responsible for experimental design of transcription profiling and analysis of the data of transcription profiling as well as manuscript preparation associated with transcription profiling. All the authors read and approved the final manuscript.

\section{Authors' information}

Harumi Jyonouchi, M.D.: She is a board certified in Pediatrics and Allergy/ Immunology and currently serves as an Associate Professor of Pediatrics, UMDNJ-NJMS. She has conducted several clinical studies addressing relationship of delayed type food allergy (FA) with Gl symptoms in ASD children as well as immune abnormalities in a subset of ASD children with distinct clinical characteristics.

Gokce A. Truner, M.D., Ph.D.: He is a molecular geneticist and currently is an Assistant Professor at the Institute of Genomic Medicine, UMDNJ-NJMS. He has been involved in clinical studies of CGH, transcription profiling, and microRNA profiling in patients with various medical conditions including children with mental retardation and ASD.

\section{Competing interests}

The authors declare that they have no competing interests.

Received: 23 August 2011 Accepted: 7 January 2012

Published: 7 January 2012

\section{References}

1. Bale TL, Baram TZ, Brown AS, Goldstein JM, Insel TR, McCarthy MM, Nemeroff CB, Reyes TM, Simerly RB, Susser ES, Nestler EJ: Early life programming and neurodevelopmental disorders. Biol Psychiatry 2010, 68:314-319.

2. Rudan I: New technologies provide insights into genetic basis of psychiatric disorders and explain their co-morbidity. Psychiatr Danub 2010, 22:190-192.

3. Toro R, Konyukh M, Delorme R, Leblond C, Chaste P, Fauchereau F, Coleman M, Leboyer M, Gillberg C, Bourgeron T: Key role for gene dosage and synaptic homeostasis in autism spectrum disorders. Trends Genet 2010, 26:363-372

4. Betancur C: Etiological heterogeneity in autism spectrum disorders: more than 100 genetic and genomic disorders and still counting. Brain Res 2011, 1380:42-77.

5. Weiss LA: Autism genetics: emerging data from genome-wide copynumber and single nucleotide polymorphism scans. Expert Rev Mol Diagn 2009, 9:795-803.

6. Ashwood P, Wills $S$, Van de Water J: The immune response in autism: a new frontier for autism research. J Leukoc Biol 2006, 80:1-15.
7. Singh VK: Phenotypic expression of autoimmune autistic disorder (AAD): a major subset of autism. Ann Clin Psychiatry 2009, 21:148-161.

8. Jyonouchi H, Geng L, Cushing-Ruby A, Quraishi H: Impact of innate immunity in a subset of children with autism spectrum disorders: a case control study. J Neuroinflammation 2008, 5:52

9. Notarangelo LD, Fischer A, Geha RS, Casanova JL, Chapel H, Conley ME, Cunningham-Rundles C, Etzioni A, Hammartrom L, Nonoyama S, et al: Primary immunodeficiencies: 2009 update. J Allergy Clin Immunol 2009, 124:1161-1178.

10. Paris K, Sorensen RU: Assessment and clinical interpretation of polysaccharide antibody responses. Ann Allergy Asthma Immunol 2007, 99:462-464.

11. Butrus S, Portela R: Ocular allergy: diagnosis and treatment. Ophthalmol Clin North Am 2005, 18:485-492, v.

12. Nassef M, Shapiro G, Casale TB: Identifying and managing rhinitis and its subtypes: allergic and nonallergic components-a consensus report and materials from the Respiratory and Allergic Disease Foundation. Curr Med Res Opin 2006, 22:2541-2548.

13. Expert Panel Report 3 (EPR-3): Guidelines for the Diagnosis and Management of Asthma-Summary Report 2007. J Allergy Clin Immunol 2007, 120:594-138

14. Alachkar H, Taubenheim N, Haeney MR, Durandy A, Arkwright PD: Memory switched $B$ cell percentage and not serum immunoglobulin concentration is associated with clinical complications in children and adults with specific antibody deficiency and common variable immunodeficiency. Clin Immunol 2006, 120:310-318.

15. Shea S, Turgay A, Carroll A, Schulz M, Orlik H, Smith I, Dunbar F: Risperidone in the treatment of disruptive behavioral symptoms in children with autistic and other pervasive developmental disorders. Pediatrics 2004, 114:e634-641.

16. Tangye SG, Cook MC, Fulcher DA: Insights into the role of STAT3 in human lymphocyte differentiation as revealed by the hyper-IgE syndrome. J Immunol 2009, 182:21-28.

17. Kainulainen L, Vuorinen T, Rantakokko-Jalava K, Osterback R, Ruuskanen O: Recurrent and persistent respiratory tract viral infections in patients with primary hypogammaglobulinemia. J Allergy Clin Immunol 2010, 126:120-126.

18. Lucas M, Lee M, Lortan J, Lopez-Granados E, Misbah S, Chapel H: Infection outcomes in patients with common variable immunodeficiency disorders: relationship to immunoglobulin therapy over 22 years. $J$ Allergy Clin Immunol 2010, 125:1354-1360 e1354.

19. Orange JS, Grossman WJ, Navickis RJ, Wilkes MM: Impact of trough IgG on pneumonia incidence in primary immunodeficiency: A meta-analysis of clinical studies. Clin Immunol 2010, 137:21-30.

20. Elenkov IJ: Neurohormonal-cytokine interactions: implications for inflammation, common human diseases and well-being. Neurochem Int 2008, 52:40-51.

21. Rivest S: Interactions between the immune and neuroendocrine systems. Prog Brain Res 2010, 181:43-53.

22. Watanabe Y, Someya T, Nawa H: Cytokine hypothesis of schizophrenia pathogenesis: evidence from human studies and animal models. Psychiatry Clin Neurosci 2010, 64:217-230.

23. Conti HR, Gaffen SL: Host responses to Candida albicans: Th17 cells and mucosal candidiasis. Microbes Infect 2010, 12:518-527.

24. Kimura A, Kishimoto T: Th17 cells in inflammation. Int Immunopharmacol 2011, 11:319-322.

25. McGeachy MJ, Cua DJ: Th17 cell differentiation: the long and winding road. Immunity 2008, 28:445-453.

26. Bousfiha A, Picard C, Boisson-Dupuis S, Zhang SY, Bustamante J, Puel A, Jouanguy E, Ailal F, El-Baghdadi J, Abel L, Casanova JL: Primary immunodeficiencies of protective immunity to primary infections. Clin Immunol 2010, 135:204-209.

27. Parihar A, Eubank TD, Doseff Al: Monocytes and macrophages regulate immunity through dynamic networks of survival and cell death. J Innate Immun 2010, 2:204-215.

28. Wiktor-Jedrzejczak W, Gordon S: Cytokine regulation of the macrophage (M phi) system studied using the colony stimulating factor-1-deficient op/op mouse. Physiol Rev 1996, 76:927-947.

29. Serbina NV, Jia T, Hohl TM, Pamer EG: Monocyte-mediated defense against microbial pathogens. Annu Rev Immunol 2008, 26:421-452. 
30. Biswas SK, Mantovani A: Macrophage plasticity and interaction with lymphocyte subsets: cancer as a paradigm. Nat Immunol 2010, 11:889-896.

31. Jurynczyk M, Selmaj K: Notch: a new player in MS mechanisms. J Neuroimmunol 2010, 218:3-11.

32. Hu X, Chung AY, Wu I, Foldi J, Chen J, Ji JD, Tateya T, Kang YJ, Han J, Gessler M, et al: Integrated regulation of Toll-like receptor responses by Notch and interferon-gamma pathways. Immunity 2008, 29:691-703.

33. Cao Q, Lu J, Kaur C, Sivakumar V, Li F, Cheah PS, Dheen ST, Ling EA: Expression of Notch-1 receptor and its ligands Jagged-1 and Delta-1 in amoeboid microglia in postnatal rat brain and murine BV-2 cells. Glia 2008, 56:1224-1237.

34. Grandbarbe L, Michelucci A, Heurtaux T, Hemmer K, Morga E, Heuschling P. Notch signaling modulates the activation of microglial cells. Glia 2007, 55:1519-1530.

35. Teachey DT, Seif AE, Brown VI, Bruno M, Bunte RM, Chang YJ, Choi JK, Fish JD, Hall J, Reid GS, et al: Targeting Notch signaling in autoimmune and lymphoproliferative disease. Blood 2008, 111:705-714.

36. Aguirre A, Rubio ME, Gallo V: Notch and EGFR pathway interaction regulates neural stem cell number and self-renewal. Nature 2010, 467:323-327.

37. Berasain C, Perugorria MJ, Latasa MU, Castillo J, Goni S, Santamaria M, Prieto J, Avila MA: The epidermal growth factor receptor: a link between inflammation and liver cancer. Exp Biol Med (Maywood) 2009, 234:713-725.

38. Sanjabi S, Zenewicz LA, Kamanaka M, Flavell RA: Anti-inflammatory and pro-inflammatory roles of TGF-beta, IL-10, and IL-22 in immunity and autoimmunity. Curr Opin Pharmacol 2009, 9:447-453.

39. DelGiudice-Asch G, Simon L, Schmeidler J, Cunningham-Rundles C, Hollander E: Brief report: a pilot open clinical trial of intravenous immunoglobulin in childhood autism. J Autism Dev Disord 1999, 29:157-160.

40. Gupta S, Samra D, Agrawal S: Adaptive and Innate Immune Responses in Autism: Rationale for Therapeutic Use of Intravenous Immunoglobulin. J Clin Immunol 2010, PMID: 20393790

41. Plioplys AV: Intravenous immunoglobulin treatment of children with autism. J Child Neurol 1998, 13:79-82.

doi:10.1186/1742-2094-9-4

Cite this article as: Jyonouchi et al:: Immunological characterization and transcription profiling of peripheral blood (PB) monocytes in children with autism spectrum disorders (ASD) and specific polysaccharide antibody deficiency (SPAD): case study. Journal of Neuroinflammation 2012 9:4.

\section{Submit your next manuscript to BioMed Central and take full advantage of:}

- Convenient online submission

- Thorough peer review

- No space constraints or color figure charges

- Immediate publication on acceptance

- Inclusion in PubMed, CAS, Scopus and Google Scholar

- Research which is freely available for redistribution 